\title{
$M-3 Y$
}

NASA Technical Memorandum 106267

1767,20

AIAA-93-3074

ICOMP-93-26

$P, 3$

\section{High Order Accurate Solutions of Viscous Problems}

M. Ehtesham Hayder

Institute for Computational Mechanics in Propulsion

Lewis Research Center

Cleveland, Ohio

and

Eli Turkel

Institute for Computational Mechanics in Propulsion

Lewis Research Center

Cleveland, Ohio

$$
\begin{aligned}
& \text { (NASA-TM-1S(267) HIGH ORDER } \\
& \text { ACCU2ATE SULUTIOUS OF VISCDUS } \\
& \text { PAOELFMS (NASA) } 13 \mathrm{P}
\end{aligned}
$$$$
\text { N94-11253 }
$$

and Tel-Aviv University

Tel-Aviv, Israel

Prepared for the 24th Fluid Dynamics Conference sponsored by the American Institute of Aeronautics and Astronautics Orlando, Florida, July 6-9, 1993

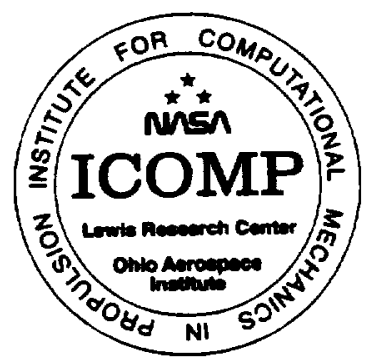





\title{
HIGH ORDER ACCURATE SOLUTIONS OF VISCOUS PROBLEMS
}

\author{
M.E. Hayder \\ Institute for Computational Mechanics in Propulsion \\ Lewis Research Center \\ Cleveland, Ohio $\mathbf{4 4 1 3 5}$ \\ E. Turkel ${ }^{*}$ \\ Institute for Computational Mechanics in Propulsion \\ Lewis Research Center \\ Cleveland, Ohio 44135 \\ and Tel-Aviv University \\ Tel-Aviv, Israel
}

\begin{abstract}
We consider a fourth order extension to MacCormack's scheme. The original extension was fourth order only for the inviscid terms but was second order for the viscous terms. We show how to modify the viscous terms so that the scheme is uniformly fourth order in the spatial derivatives. Applications are given to some boundary layer flows. In addition, for applications to shear flows the effect of the outflow boundary conditions are very important. We compare the accuracy of several of these different boundary conditions for both boundary layer and shear flows. Stretching at the outflow usually increases the oscillations in the numerical solution but the addition of a filtered sponge layer (with or without stretching) reduces such oscillations. The oscillations are generated by insufficient resolution of the shear layer. When the shear layer is sufficiently resolved then oscillations are not generated and there is less of a need for a nonreflecting boundary condition.
\end{abstract}

\section{Basic Scheme}

We consider the equation

$$
u_{t}+f_{x}=\left(b(x) u_{x}\right)_{x}
$$

The original scheme suggested by Gottlieb and Turkel [5] was

- Professor,Department of Mathematies, member AIA A Copyright @1993 American Institute of Aeroneutics and Astronauties, Inc. No copyright is asserted in the United States under Title 17, U.S. Code. The U.S. Government has a royalty-free license to exercise all rights under the copyright claimed herein for Governmentel purposes. All other rights are reserved by the copyright owner.

$$
u^{(1)}=u^{n}+\frac{\Delta t}{6 \Delta x}\left(-F_{i+2}^{n}+8 F_{i+1}^{n}-7 F_{i}^{n}\right)
$$

where

$$
F_{i}^{n}=\left[-f_{i}+b_{i} \frac{\left(u_{i}-u_{i-1}\right)}{\Delta x}\right]^{n}
$$

with a second stage

$$
\begin{gathered}
u_{i}^{n+1}=\frac{u_{i}^{n}+u_{i}^{(1)}}{2}+\frac{\Delta t}{12 \Delta x}\left(7 F_{i}^{(1)}-8 F_{i-1}^{(1)}+F_{i-2}^{(1)}\right) \\
F_{i}^{(1)}=\left[-f_{i}+b_{i} \frac{\left(u_{i+1}-u_{i}\right)}{\Delta x}\right]^{(1)}
\end{gathered}
$$

This scheme is second order in time and third order in space if $b(x)=0$, but is only second order in space for the viscous term. We label the above scheme FB since it has a forward difference on the first stage and a backward difference in the second stage. We next present the BF variant as

$$
\begin{gathered}
u^{(1)}=u^{n}+\frac{\Delta t}{6 \Delta x}\left(F_{i-2}^{n}-8 F_{i-1}^{n}+7 F_{i}^{n}\right) \\
F_{i}^{n}=\left[-f_{i}+b_{i} \frac{\left(u_{i+1}-u_{i}\right)}{\Delta x}\right]^{n}
\end{gathered}
$$

with a second stage

$$
u_{i}^{n+1}=\frac{u_{i}^{n}+u_{i}^{(1)}}{2}-\frac{\Delta t}{12 \Delta x}\left(7 F_{i}^{(1)}-8 F_{i+1}^{(1)}+F_{i+2}^{(1)}\right)
$$

where

$$
F_{i}^{(1)}=\left[-f_{i}+b_{i} \frac{\left(u_{i}-u_{i-1}\right)}{\Delta x}\right]^{(1)}
$$

It is shown in [5] that by rotating these two variants (i.e. alternating the order of the sweeps) 
one can obtain fourth order accuracy in space when $b(x)=0$. However, one still obtains only second order accuracy for the viscous terms. When the Reynolds number is large then the dominant error comes from the inviscid portion and the total error is essentially fourth order in space. However, there are cases when one wishes the spatial error to be uniformly fourth order. We propose to accomplish this by a small modification to (2). The forward step is modified to :

$$
F_{i}^{n}=\left[-f_{i}+\frac{1}{6}\left(-b_{i}+8 b_{i-1}-b_{i-2}\right) \frac{\left(u_{i}-u_{i-1}\right)}{\Delta x}\right]^{n}
$$

A similar modification is used on the backward differences of $F$. The stencil is now widened in that point $i$ in the predictor requires a centered five point stencil as does the corrector. This wider stencil occurs only because of the coefficient $b$. If $b$ is a function only of $x$ then this extended stencil is not of any importance. For the Navier-Stokes equations the function $b$ is essentially the viscosity $\mu$. If one uses Sutherland's law to compute $\mu$ then $b$ is a nonlinear function and so one needs to store data in artificial cells for both the predictor and corrector at all boundaries. Thus, the way that the scheme is now used is: update the solution at all interior points. Use third order extrapolation to define the fluxes at two artificial points outside all the boundaries (see [1]). Apply the corrector at all interior points and then again extrapolate to the artificial points. At all solid boundaries the boundary conditions are imposed before the extrapolation. We stress that the extrapolation of the fluxes is identical to using one sided differences at the next update. The use of extrapolation rather than one sided differences is done only to improve the vectorization of the algorithm. A Taylor series expansion verifies that after rotating between the two variants the scheme is now fourth order accurate for both the hyperbolic and parabolic portions of the scheme.

\section{Boundary Conditions}

One of the main difficulties in solving the NavierStokes equations is the treatment of the outflow boundary condition. We are mainly interested in the viscous but high Reynolds number flow. The first question is whether the boundary treatment should be based on the Euler equations or the NavierStokes equations. The difference between the two approaches is not just the type of boundary conditions but even the number of boundary conditions that needs to be externally given. For inviscid flow, when the flow is subsonic one boundary condition needs to be specified corresponding to an incoming acoustic wave. For supersonic flow no boundary conditions are specified. For viscous the flow the equations are no longer hyperbolic but rather incompletely parabolic. For subsonic flow four conditions need to be specified (in two dimensions), while for supersonic flow three conditions need to be specified. Moreover, for the inviscid (hyperbolic) case the needed boundary conditions should be of Dirichlet type, i.e. a combination of the dependent variables is specified. For the viscous problem (parabolic) the specified boundary condition can be either of Dirichlet type or Neumann type (combinations of normal derivatives) or a combination of both of these (Robin type). Many codes use inviscid type boundary conditions. This is based on the assumption that the flow in the far field is essentially inviscid because of the high Reynolds number and the lack of physical boundary layers.

To be more specific we need to consider different types of configurations. For boundary layer flows one needs to distinguish between the portion of the computational domain inside and outside the boundary layer. Outside the boundary layer one may be able to use inviscid type boundary conditions. Inside the boundary layer the pressure should be specified. Gustaffson [7] has shown that the problem is also well posed if one extrapolates all the variables inside the boundary layer. For external flow about an airfoil some codes use inviscid type conditions while others extrapolate all the variables. Though the flow seems to be inviscid in the far field nevertheless viscous effects persist in the wake region. Thus, for example, there is a velocity defect no matter how far one goes downstream and the integral of this is constant. Thus, as one proceeds further downstream the defect locally gets smaller but is spread over a larger region. This should affect the appropriate boundary conditions. In this paper we will also consider free shear flows. Here too viscous effects should be important near the shear layer even far downstream. A further complication that is most pronounced for shear flows is that one does not know the solution downstream and therefore one cannot impose any type of Dirichlet boundary condition. Frequently, there is a significant spreading of the shear layer and so one does not know in advance even where the shear layer will intersect the outflow boundary. Furthermore, many theories expand the solution about a constant pressure in the far field and so obtain a differential equation for the pressure deviation. For shear flows the pressure differs on the two sides of the shear and so the pressure is not con- 
stant in the far field except if the far field boundary is extremely far away which is not computationally practical. In other words, some of the nonreflecting boundary conditions that have been proposed in the literature are based on suppositions of the form of the outgoing wave, e.g. a plane wave or a spherical wave. However, these assumptions are not valid for shear flows.

In spite of all these dangers we shall consider characteristic-like boundary conditions at outflow and so the number of boundary conditions will be given by the inviscid theory. Nevertheless we shall see that viscous effects are at least partially accounted for.

1. BC1: The simplest approach is to freeze the characteristic variables normal to the surface and to specify the incoming characteristic variable and to extrapolate the outgoing variables. For a one dimensional hyperbolic system one can show that such a procedure is well posed [6]. This approach was used by many authors in the past.

2. BC2: An improved version of this system is to use differential equations that correspond to these characteristic variables. Thus, for the acoustic waves one needs differential equations for $p_{t} \pm \rho c u_{t}$ where $\mathrm{u}$ is the velocity component normal to the boundary. For the shear wave we need $v_{t}$ where $\mathrm{v}$ is tangential to the boundary and finally $p_{t}-c^{2} \rho_{t}$ for the entropy variable. Whenever the boundary condition is not specified but free to float then the appropriate characteristic variable is updated by the partial differential equation. In order to avoid one sided differences the fluxes are extrapolated outside the domain to artificial points. Whenever the appropriate combination is specified then we replace this by specifying the combination of the time derivatives. We can describe this as

$$
\begin{gathered}
p_{t}-\rho c u_{t}=R_{1} \\
p_{t}+\rho c u_{t}=R_{2} \\
p_{t}-c^{2} \rho_{t}=R_{3} \\
v_{t}=R_{4}
\end{gathered}
$$

where $R_{i}$ is determined by which variables are specified and which are not. Whenever, the combination is not specified that $R_{i}$ is just those spatial derivatives that come from the Navier-Stokes equations. Thus, $R_{i}$ contains viscous contributions even though the basic format is based on inviscid characteristic theory. In implementing these differential equations we convert them to conservation variables $\rho, m=\rho u, n=\rho v, E$. Assuming an ideal gas we then have

$$
\begin{gathered}
p_{t}=(\gamma-1)\left(E_{t}+\frac{u^{2}+v^{2}}{2} \rho_{t}-u m_{t}-v n_{t}\right) \\
u_{t}=\frac{m_{t}}{\rho}-\frac{u \rho_{t}}{\rho} \\
v_{t}=\frac{n_{t}}{\rho}-\frac{v \rho_{t}}{\rho}
\end{gathered}
$$

For subsonic flow, the immediate generalization of the first method is to set $R_{1}=0$ and to calculate $R_{2}, R_{3}, R_{4}$ from the NavierStokes equations.

3. BC3: We next consider improvements for the last boundary condition. These improvements all leave $R_{2}, R_{3}, R_{4}$ as before from the Navier-Stokes equations. The changes are all to the incoming wave, $R_{1}$. Giles [4] (and later Kroner [10]) added some $y$ (tangential) space derivatives to the first equation. Thus the equation for $R_{1}$ is replaced by

$$
p_{t}-\rho c u_{t}+u \rho c v_{y}+v\left(p_{y}-\rho c u_{y}\right)=0
$$

As before all the derivatives can be transformed to derivatives of conservation variables.

4. BC4: Based on an asymptotic expansion of the wave equation Bayliss and Turkel [2] derived the following nonreflecting condition to replace $R_{1}$.

Define $d^{2}=\frac{x^{2}}{1-M^{2}}+y^{2}$ where $M$ is the Mach number. $x, y$ are the physical locations of the boundary point relative to some source, usually the inflow. Then

$$
\begin{gathered}
p_{t}-\rho c \frac{x}{d \sqrt{1-M^{2}}}\left(u_{t}-u v_{y}\right) \\
+c \sqrt{1-M^{2}} \frac{y}{d} p_{y}+\frac{c \sqrt{1-M^{2}}}{2 d}\left(p-p_{\infty}\right) \\
=0
\end{gathered}
$$

As stated before one frequently does not know $p_{\infty}$ and so we shall simply ignore the last term in this equation.

We note that this equation does not have the form of the first equation for $R_{1}$, i.e. it is not an equation for a characteristic variable. 
5. BC5: For many cases the domain is much longer than it is high. In this case we can ignore $y$ relative to $x$. Hence, we assume $\frac{x}{d} \sim$ $\sqrt{1-M^{2}}, \frac{y}{d} \sim 0$. Then (3) simplifies to

$$
p_{t}-\rho c\left(u_{t}-u v_{y}\right)=0
$$

With this simplification we get an equation for the characteristic variable in a form similar to that proposed by Giles but not identical with his condition.

Besides nonreflecting boundary conditions based on the partial differential equation there are other approaches to removing the difficulties associated with far field boundaries. One popular approach is to use a 'sponge layer'. The idea behind this approach is to introduce a domain between the region of interest and the actual outflow boundary. In this region one changes the differential equation in some manner so as dissipate the energy of the system or else prevent waves from traveling back into the domain. Since, the equations have been changed the solution in this sponge layer has no physical relevance. Hopefully, the solution in the rest of the domain is physically relevant and is not contaminated with any false reflections from the boundary or the sponge layer itself. In this approach the boundary condition at the outflow boundary is irrelevant. It may introduce perturbations but these are eliminated in the sponge layer. For this approach to be effective the sponge layer must be small relative to the rest of the domain. A sponge layer approach suggested by Colonius et. al [3] is to stretch the grid and then filter the solution near the outflow boundary. They used stretching throughout the domain and applied filter near the outflow (in the sponge layer). Karni [9] used sponge layer with modified governing equations to accelerate convergence to steady state.

\section{Results}

In this section we check on the improvements to the 2-4 scheme presented above. We consider flow over a flat plate with $M=0.25, R e=100$ and $P r=0.72$. We use a uniform cartesian mesh in both the directions. In figure 1 we compare the original method which is fourth order accurate only for the inviscid terms but second order for the viscous part with the improved method which is uniformly fourth order accurate. In this figure we plot the $u$ component of the velocity versus the normalized $y$ distance $(\eta)$. We consider two meshes. The finer mesh is $400 \times 80$ and the coarser mesh is $200 \times 40$. On the finer mesh both the original scheme and the improved method give similar results. On the coarser mesh we clearly see the improvement that comes from using a fourth order accurate treatment of the viscous terms.

We next consider a shear flow [8] modelling a planar jet. All the boundary conditions used in this study are based on the assumption of an outgoing plane wave expansion or else a circular wave expansion. Neither of these assumptions is valid for a jet geometry. Jet acoustics presents an additional difficulty for far field boundaries. The standard assumption is that the further out the artifcial boundary is, the more accurate the results are since it better simulates an infinite domain. However, in many cases the flow is spatially unstable and as the length of the domain in the $x$ dimension gets larger, the waves become more unstable and grow until nonlinear behavior either saturates the wave growth or else the code stops running because of an explosive nonlinear growth. Nevertheless, we shall use these boundary conditions as the best available. The basic mesh is uniform in the $x$ direction and stretched in the $y$ direction. The size of the standard domain is $100 \times 5$ with $600 \times 60$ grid points. We also consider the addition of a layer beyond $x=100$. This additional grid which we call a sponge layer is just 60 additional grid points beyond $x=100$ until $x=133$ with a stretched mesh. We use a stretching proportional to $x^{1.5}$. For ease of comparison all figures use the same scaling and show 160 radii in the axial and 5 radii in transverse direction respectively. The figures are shown after a nondimensional time of 500 which requires about a hundred thousand time steps. The solution should be approaching a steady state.

We now consider a case where both the inner and outer flows are subsonic, with the jet at $M=0.8$ and the outer flow at $M=0.28$. Hence, the nonreflecting boundary conditions are applied along the entire outflow line. The Reynolds number for this flow is 10,200 based on the (planar) jet radius. We compare the different boundary conditions and the effect of sponge layer with and without filtering. Figure 2a shows the contour plot of the vorticity for the standard case with a finer mesh in the $y$ direction and an extended, though uniform, domain in the $x$ direction, so that the mesh is now $900 \times 100$ and $x \leq 150$. We do not have a way of quantitatively comparing these solutions. Hence, we shall content ourselves with qualitatively comparing the solutions with this finer grid solution. We now consider the outflow boundary conditions BC2 - BC5 respectively. In figures $2 b-2 e$ we plot the vorticity for the same physical case as before but with 
the standard mesh, i.e. $600 \times 60$ and $x \leq 100$. The stretching in the $\mathrm{y}$ direction is stronger in the finer mesh (figure $2 \mathrm{a}$ case) than the standard mesh (fig. 2b-2e). All the boundary conditions on the same mesh give similar solutions. Note, that in all these cases the vorticity is visible much closer to the inflow than when the outer boundary was at $x=150$. This demonstrates that for jet flows the position of the outer boundary is more important that the details of the nonreflecting boundary condition. Among the various boundary conditions the condition $\mathrm{BC} 2$ is slightly worse than the others. It is our observation that the mesh density in the $y$ direction has a greater effect than the difference between the various boundary conditions.

We next consider the introduction of the sponge layer with the nonuniform increasing grid. In this layer we add 60 grid points at the outflow boundary to the previous 600 points. The mesh in the sponge layer is stretched in the $x$ direction using the stretching function $x^{1.5}$. Stretching in $y$ is the same as the previous $600 \times 60$ grid cases. The physical mesh is now $x \leq 150$. Because of this stretching the resolution of the vortices near the outer boundary is severely reduced. Now, the effect of the different boundary conditions is negligible. Hence, in figure 3 we plot the results obtained with the Giles boundary condition. The vortex growth is slightly delayed compared with figure 2.3 while the vortices near $x=100$ are stronger. As expected the vortices near the new outer boundary are washed out.

We next introduce the filter given below in last 160 points near the outflow boundary, i.e. the 60 points of the sponge layer and an additional 100 interior points. The filter is given by

$\bar{f}_{i}=.625 f_{i}+.25\left(f_{i+1}+f_{i-1}\right)-.0625\left(f_{i+2}+f_{i-2}\right)$

This filter is 4 th order accurate for a uniform mesh. The filter function used by Colonius et. al. [3] has a similar form but variable coefficients. We plot the vorticity field for this case in figure 4. Again, the numerical solution is independent of the far field boundary condition and so in figure 4 we only consider the case using the Giles boundary condition. We see that the stretched mesh coupled with a larger domain delays the growth of the vorticities. Hence, the beginning of the vortex growth is closer to figure $2 \mathrm{a}$ than the previous cases. In the far field the vortex growth has been completely destroyed by the filtering. Hence, we only expect accurate solutions for $x \leq 100$.

We finally consider a flow with the jet entering at $M=1.5$ while the outer region has an inflow of $\mathrm{M}=0.53$. Since the inner region has a super- sonic flow at the exit all the variables are extrapolated. The nonreflecting boundary conditions are used only in the outer region. The Reynolds number based on the jet radius is about $6.37 \times 10^{3}$. In figure $5 \mathrm{a}$ we consider the fine resolution grid of $900 \times 100$ as in the subsonic case. In this case there is a much lower growth rate than in the subsonic case and the vortices are barely forming at $x=150$. In figures $5 b-5 e$, we compare the solutions of the flow computed with the various outflow boundary conditions $\mathrm{BC2}-5$. If we reduced the mesh in the $y$ direction to 60 points, as in the subsonic case, then the mesh is not fine enough to allow for vortex growth. Hence, for the smaller domain we shall consider a mesh of $600 \times 100$ with $x \leq 100$. In this mesh we use the same stretching in the $y$ direction as in the subsonic cases (fig. 2b-2e). In this case the Giles boundary condition seems to be slightly worse than the others and generates more, false, vorticity. On the other hand if we add a sponge layer with a stretched mesh, as before, the only reasonable solutions are given by the Giles boundary condition. Adding the filter, as given above, eliminated all the vortices and the solution is essentially independent of the outer boundary treatment. This is the best solution but is special for this case. Hence, the supersonic case is less useful for comparing treatments of the outer boundary. All the boundary conditions considered here are based on the inviscid case even though we compute the full Navier-Stokes equations. Thus, we assume that the viscosity is negligible at the outflow.

\section{Conclusions}

We have shown how to modify the fourth order extension of MacCormack's scheme so that it is uniformly fourth order accurate for both the inviscid and viscous portions of the flux. This results in increased accuracy for boundary layer type flows at local unit Reynolds numbers of 1000 and lower.

We next compared several boundary conditions for jet flow. In all cases it is best to use the partial differential equations at the outflow boundary itself. This can be accomplished by either using one sided differences or else some extrapolation to artificial points beyond the boundary. This extrapolation is equivalent to a one sided difference formula within the differential operator solver. One then takes combinations of these updated differences together with a radiation boundary condition to form the final updated solution. The combination of given boundary conditions and equation solvers is determined by characteristic theory for the inviscid portion of the system. Nevertheless, 
updating the complete equations introduces some viscous information into the procedure.

Though the differences were not large, the best results were obtained with the radiation boundary condition of Giles or else the simplification of the boundary condition of Bayliss and Turkel. An alternative is to stretch the mesh at the outflow and then to use the obtained solution only in the original domain. This requires extra storage and computer time but yields somewhat better solutions. In this case all the boundary treatments at the outflow boundary give essentially identical results as the major effect is the stretching of the mesh. Introduction of a filter in this sponge layer with the expanding mesh smooths out all significant features of the solution in the far field.

\section{References}

[1] Bayliss, A. Maestrello, L. and Turkel, E. $A$ Fourth Order Method for the Unsteady Compressible Navier-Stokes Equations, AIAA paper 85-1694, 1985.

[2] Bayliss, A. and Turkel, E. Far Field Boundary Condition for Compressible Flows, Journal Comput. Physics, 48, 1982, pp. 182-199.

[3] Colonius, T., Lele, S. K. and Moin, P., Boundary Conditions for Direct Computations of Aerodynamic Sound Generation, AIAA paper 92-02-075.

[4] Giles, M.G., Nonreflecting Boundary Conditions for Euler Equations Calculations, AIAA Journal, 1990, Vol 28, pp. 2050-2058.

[5] Gottlieb, D. and Turkel, E. , Dissipative TwoFour Methods for Time Dependent Problems, Math. Comput., 30, 1976, pp. 703-723.

[6] Gottlieb, D., Gunzburger, M. and Turkel, E., On Numerical Boundary Treatment for Hyperbolic Systems for Finite Difference and Finite Element Methods, SIAM Journal on Numerical Analysis, 19, 1982, pp. 671-682.

[7] Gustaffson, B. and Nordstrom, J., Extrapolation Procedures at outflow Boundaries for the Navier-stokes equations Comp. Meth. in Appl. Sciences and Engineering, Editor Glowinski and Lichnewsky, SIAM Publishers, 1990, pp. 136-150.

[8] Hayder, M.E. Turkel, E. and Mankbadi, R. R., Numerical Simulations of a High Mach Number Jet Flow, AIAA paper 93-0653, 1993.
[9] Karni, S., Accelerated convergence to SteadyState by Gradual Far-Field Damping, AlAA J., Vol. 30, pp. 1220-1228, 1992 .

[10] Kroner, D., Absorbing Boundary Conditions for the Linearized Euler Equations in 2-D, Math. Comput. 57, 1991, pp. 153-167. 


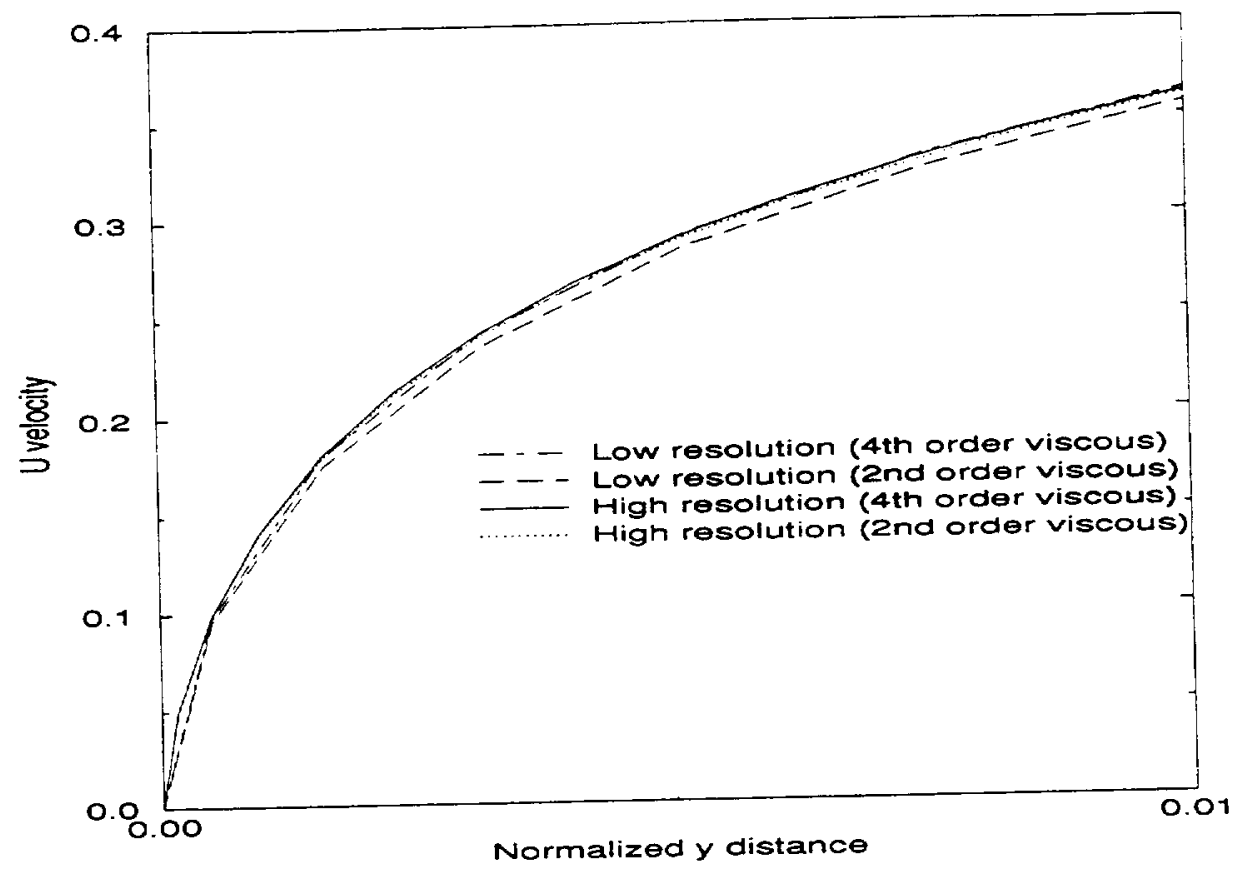

Figure 1: Boundary layer flow with $\mathrm{Re}=100$ for second and fourth order versions and two meshes

VORTICITY MAGNTIUDE

$-$

Figure 2a: Subsonic jet simulation with high resolution in large domain 


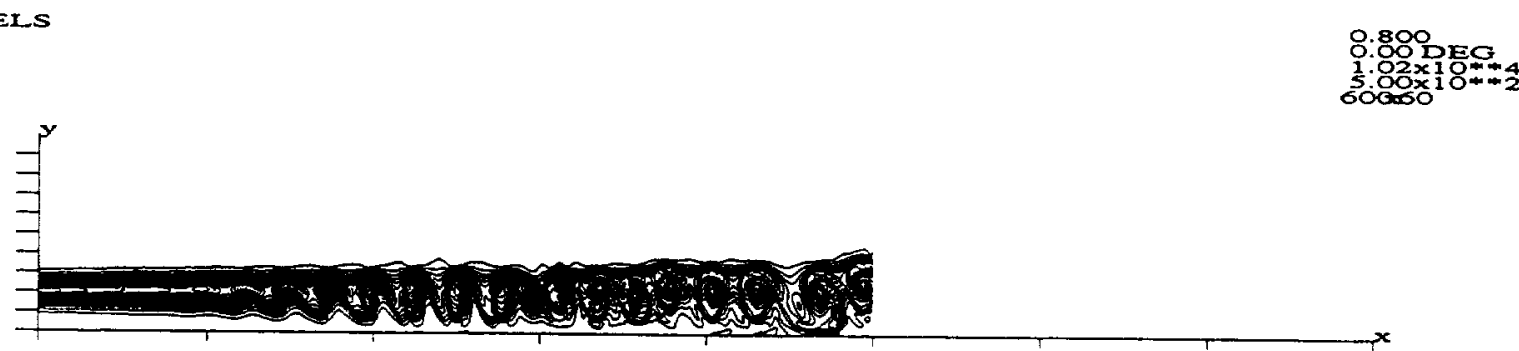

MACH

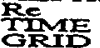

Figure 2b: Subsonic jet with BC2 boundary condition

VORTICTTY MAGNTTUDE
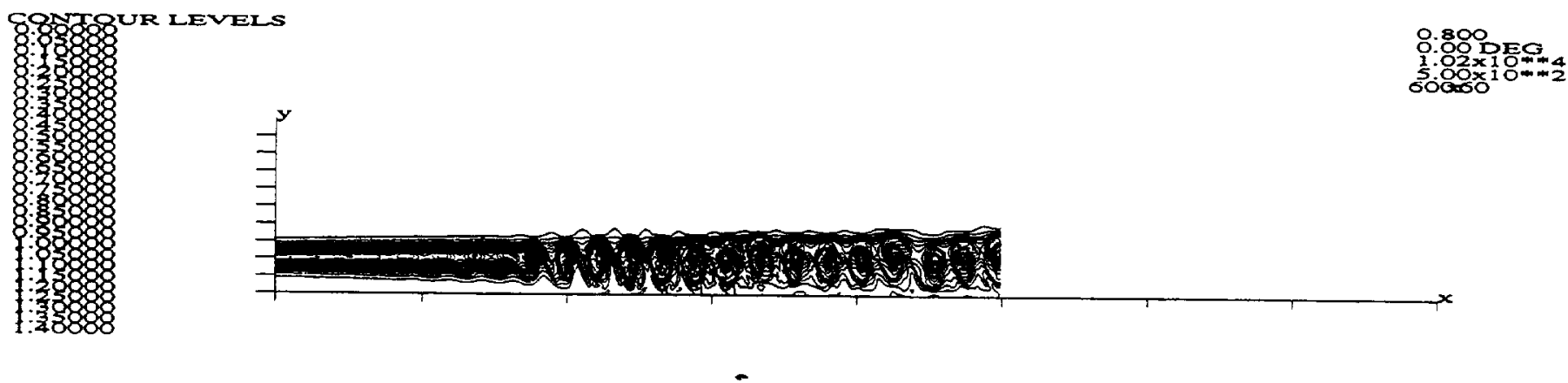

Figure 2c: Subsonic jet with Giles' boundary condition

VORTICTTY MAGNTTUDE
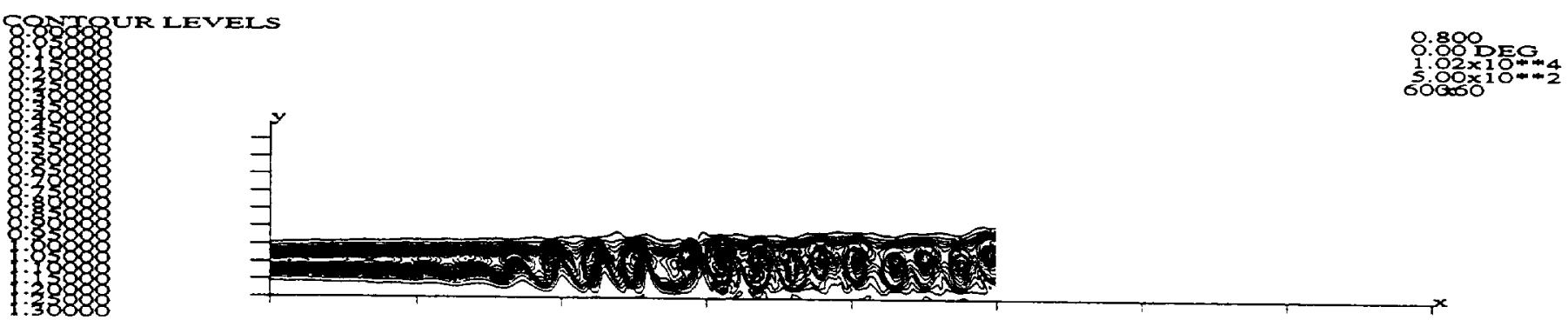

MACHA

Figure 2d: Subsonic jet with Bayliss-Turkel boundary condition 


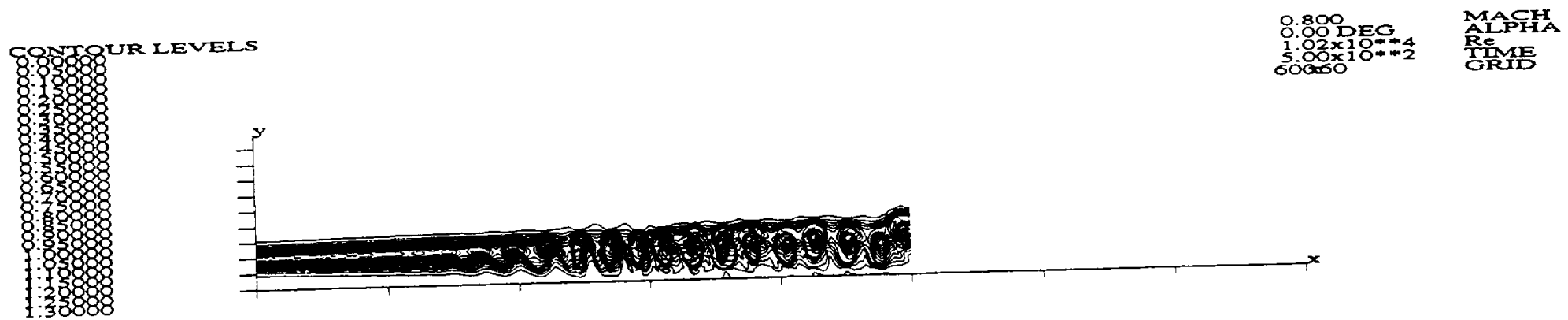

Figure 2e: Subsonic jet with simplified Bayliss-Turkel condition

Figure 2: Comparison of boundary treatments for subsonic flows

VORTICTIY MAONITUDE

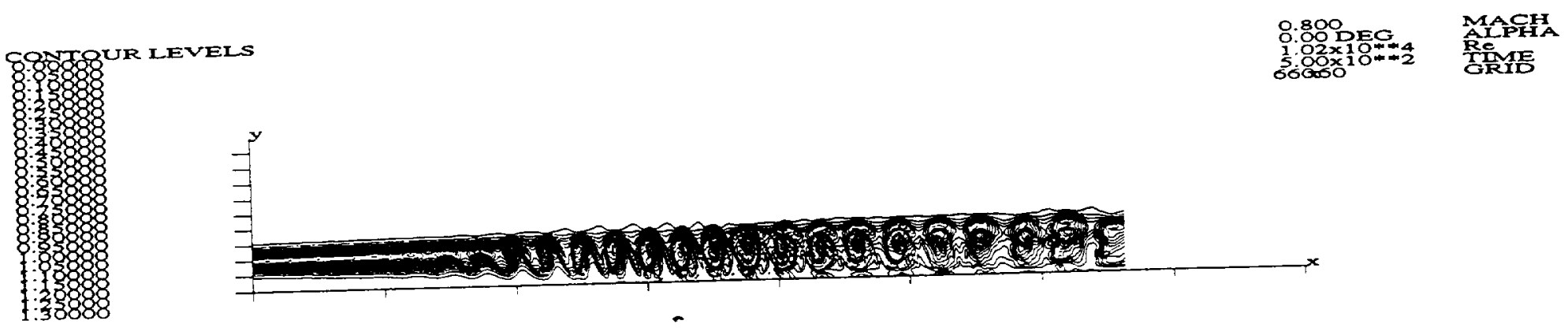

Figure 3: Subsonic jet with sponge layer and without filter

VORTTCTIT MAGNTTUDE

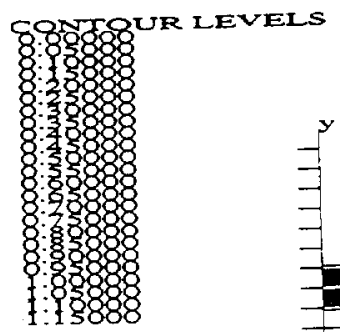

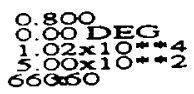

MACHA

RTID

फ)

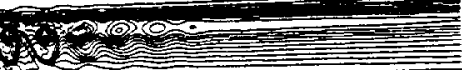

$+x$

Figure 4: Subsonic jet with sponge layer and filter 


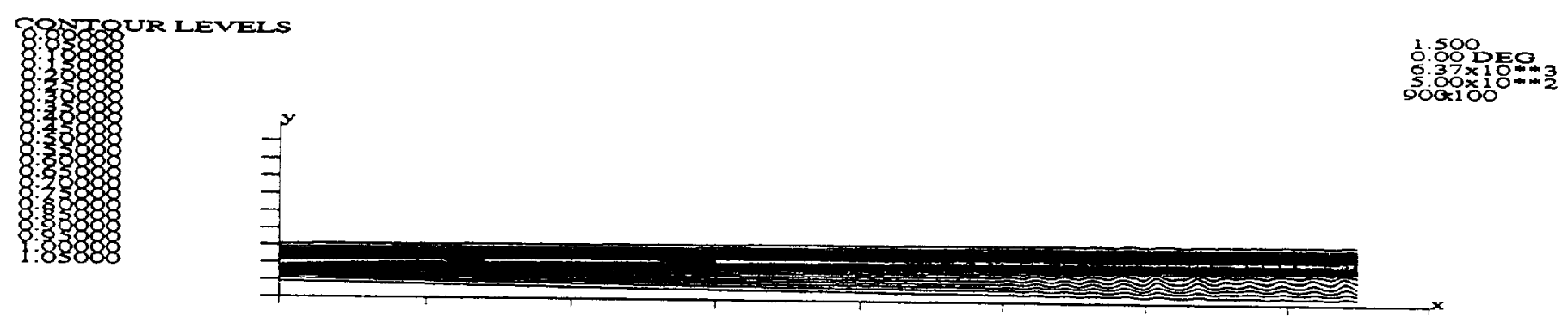

Figure 5a: Supersonic jet simulation with high resolution in large domain

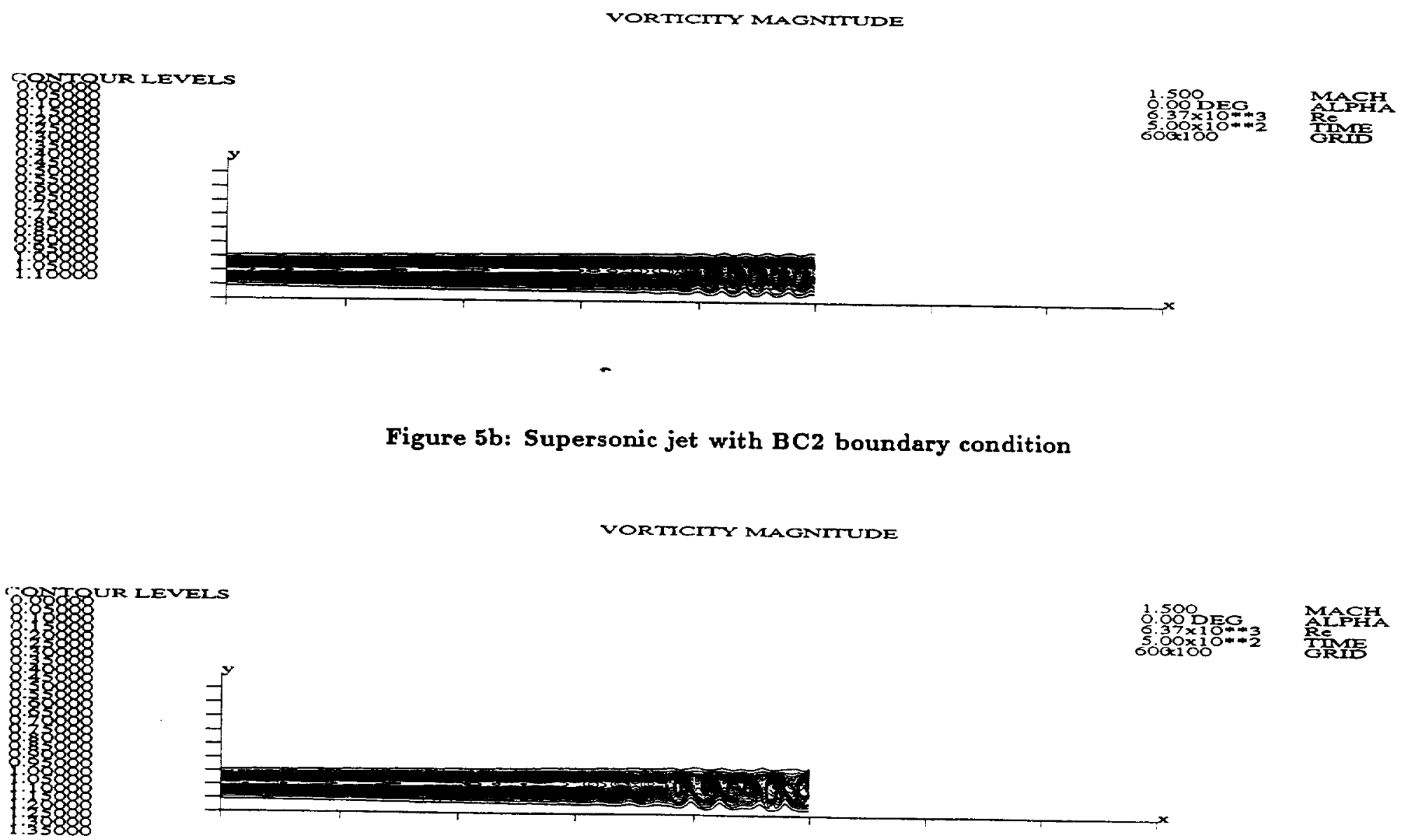

Figure 5c: Supersonic jet with Giles' boundary condition 
Public reporting burden for this collection of information is estimated to average 1 hour per response, including the time for reviewing instructions, searching existing data sources, gathering and maintaining the data needed, and completing and reviewing the collection of information. Send comments regarding this burden estimate or any other aspect of this collection of iniomation, including suggestions for recucing this burden, to Washington Headquarters Services, Directorate for Information Operations and Reports, 1215 Jefferson Davis Highway, Suite 1204, Arlington, VA 22202-4302, and to the Office of Management and Budget, Papenwork Reduction Project (0704-0188), Washington, DC 20503.

\begin{tabular}{|l|c|c|}
\hline 1. AGENCY USE ONLY (Leave blank) & $\begin{array}{c}\text { 2. REPORT DATE } \\
\text { July } 1993\end{array}$ & $\begin{array}{r}\text { 3. REPORT TYPE AND DATES COVERED } \\
\text { Technical Memorandum }\end{array}$
\end{tabular}

4. TIILEAND SUBTIILE

High Order Accurate Solutions of Viscous Problems
5. FUNDING NUMBERS

WU-505-62-21

\section{AUTHOR(S)}

M. Ehtesham Hayder and Eli Turkel

\section{Performing organization name(S) AND address(ES)}

National Aeronautics and Space Administration

Lewis Research Center

Cleveland, Ohio 44135-3191
8. Performing organization REPORT NUMBER

E-7994

\section{SPONSORING/MONITORING AGENCY NAME(S) AND ADDRESS(ES)}

National Aeronautics and Space Administration Washington, D.C. 20546-0001
10. SPONSORING/MONTTORING AGENCY REPORT NUMBER

NASA TM-106267

AIAA-93-3074

ICOMP-93-26

\section{SUPPLEMENTARY NOTES}

Prepared for the 24th Fluid Dynamics Conference sponsored by the American Institute of Aeronautics and Astronautics, Orlando, Florida, July 6-9, 1993. M. Ehtesham Hayder, Institute for Computational Mechanics in Propulsion, NASA Lewis Research Center, (work funded under NASA Cooperative Agreement NCC3-233); and Eli Turkel, Institute for Computational Mechanics in Propulsion, NASA Lewis Research Center, and Tel-Aviv University, Department of Mathematics, Tel-Aviv 69978, Israel. ICOMP Program Director, Louis A. Povinelli, (216) 433-5818.

12a. DISTRIBUTION/AVAILABILTY STATEMENT

Unclassified - Unlimited

Subject Categories 34 and 64

\section{ABSTRACT (Maximum 200 words)}

We consider a fourth order extension to MacCormack's scheme. The original extension was fourth order only for the inviscid terms but was second order for the viscous terms. We show how to modify the viscous terms so that the scheme is uniformly fourth order in the spatial derivatives. Applications are given to some boundary layer flows. In addition, for applications to shear flows the effect of the outflow boundary conditions are very important. We compare the accuracy of several of these different boundary conditions for both boundary layer and shear flows. Stretching at the outflow usually increases the oscillations in the numerical solution but the addition of a filtered sponge layer (with or without stretching) reduces such oscillations. The oscillations are generated by insufficient resolution of the shear layer. When the shear layer is sufficiently resolved then oscillations are not generated and there is less of a need for a nonreflecting boundary condition.

\section{SUBJECT TERMS}

High order algorithm; Nonreflecting boundary condition

\begin{tabular}{|l|c|}
\hline $\begin{array}{l}\text { 17. SECURITY CLASSIFICATION } \\
\text { OF REPORT } \\
\text { Unclassified }\end{array}$ & $\begin{array}{c}\text { 18. SECURITY CLASSIFICATION } \\
\text { OF THIS PAGE } \\
\text { Unclassified }\end{array}$ \\
\hline
\end{tabular}

19. SECURTY CLASSIFICATION OF ABSTRACT Unclassified 